DE DE GRUYTER

OPEN

G

BULGARIAN ACADEMY OF SCIENCES

CYBERNETICS AND INFORMATION TECHNOLOGIES • Volume 14, No 2

Sofia • 2014

Print ISSN: 1311-9702; Online ISSN: 1314-4081

DOI: $10.2478 /$ cait-2014-0026

\title{
Adaptive Sliding Mode Control Based on a Filter for Four-Wheel Omni-Directional Mobile Robots
}

\author{
Jianping Chen*, Jianbin Wang**, Sijie Ouyang***, Yimin Yang** \\ * School of Computer Science, Zhaoqing University, Zhaoqing 526061, Guangdong, China \\ ** School of Automation, Guangdong University of Technology, Guangzhou 510090, China \\ ***School of Electronic Information and Mechnical \& Electrical Engineering, Zhaoqing University, \\ Zhaoqing 526061, Guangdong, China \\ Emails: jpchen@zqu.edu.cn wangjianbin505@163.com oysjjj@zqu.edu.cn yiyang@gdut.edu.cn
}

\begin{abstract}
Since there are usually parameter uncertainties and influence of the exogenous disturbances on the dynamic model of a four-wheel omni-directional mobile robot (FOMR), the traditional strategy for motion control has not good performance. A sliding mode control based on an adaptive approach and a filter (AFSMC) is presented in this paper. First, according to identifying the reaching gain by a Radial Basis Function based neural network, and combining a filter, AFSMC can reduce the inherent impact that is produced by the normal sliding mode control. Second, the adaptive approach is applied to deal with the uncertainties and the influence of exogenous disturbances. Numerical simulations are carried out to assess the performance of the controller. All the simulation results indicate that the proposed control strategy is efficient to solve the problem.
\end{abstract}

Keywords: Four-wheel drive, omni-directional mobile robot, adaptive sliding mode, filter.

\section{Introduction}

The omni-directional mobile robot can move in any direction without changing any position and posture. The omni-directional mobile robot has been widely applied to human production and life practice in recent years due to the special motion 
advantages. The control problems of the motion and regulation have been extensively studied and have attracted the interest of many control researchers in the field of omni-directional mobile robotics [1-3].

As usual, it is common the motion control problems of the omni-directional mobile robotics to be addressed, taking into account the kinematical equations [4]. Considering its kinematical model only, several control strategies have been proposed. A back-stepping controller with global stability based on Newton mechanics model is designed in [5]. In [6] the authors have solved the motion control problem with a nonlinear back-stepping controller for a three-wheel omnidirectional mobile robot and the control values have been optimized by the sum of squares technology. The studies above mentioned are based on neglecting the uncertainties of the model and the assumption that the robot is working in ideal conditions. However, good motion control for a mobile robot with its kinematical model needs to track the designed velocity perfectly, which is impossible in a practical application. That is why a number of contributions have been focused on the dynamic representation of the omni-directional mobile robot [7, 8]. For the trajectory tracking control of an omni-directional wheeled robot for lower limbs rehabilitative training, in [9] the control problem and the interference rejection are translated into L2 control design problem, and a tracking controller is presented considering the back-stepping strategy. In the same aspect, a dynamic model of the mobile robot is considered in order to study the slipping effects between the wheels of the vehicle and the working surface [10]. In order to deal with the parameter uncertainties and the influence of the exogenous disturbances existing in the dynamic model, a sliding mode variable structure approach is presented [11-14]. In [12], based on the linearized system, an integral sliding mode control is designed for trajectory tracking control of an omni-directional mobile robot. But it has inherent deficiency, which needs computing the upper boundness of the system dynamics and may cause high noise amplification. A robust neural network NNbased sliding mode controller, which uses an NN to identify the unstructured system dynamics directly, is further proposed to overcome the disadvantages of the integral sliding mode control in [12] and reduce the online computing burden of the conventional NN adaptive controllers [13]. A path tracking control method based on an adaptive approach and neural dynamics for a wheeled mobile robot is presented, which can ensure the robot velocity asymptotically approaching to the desired velocity in uncertain system dynamics [14]. However, this implies computing more parameter values and can also cause high control costs.

In order to deal with model uncertainties and exogenous disturbances in the dynamic equations of a four-wheel omni-directional mobile robot, a variable structure control based on an adaptive approach and a filter is presented. The stability of the closed-loop system and the convergence of the adapting process are strictly demonstrated by Lyapunov stability theory. The simulation results show that this method has good tracking robustness and high control precision, simple achievement and efficiently eliminated buffeting. 


\section{Dynamic model of a four-wheel omni-directional mobile robot}

Four-wheel robots are of the type robots models, which are used in many domains. They are omni-directional with four wheels and have the ability to move in any direction at any time (they are holonomic mobile robots, in other words). Fig.1 shows the scheme of a four-wheel robot, the angles and directions of the four wheels.

\subsection{A kinematic model}

According to the geometric relationship of Fig. 1, the robot posture (position and orientation) in the robot coordinate frame is expressed as $X_{\mathrm{m}}=\left(\begin{array}{lll}x & y & \theta\end{array}\right)^{\mathrm{T}}$, and the robot posture can be presented in the world coordinate frame as $X_{\mathrm{w}}=\left(\begin{array}{lll}X & Y & \theta\end{array}\right)^{\mathrm{T}}$, the relationship between $X_{\mathrm{m}}$ and $X_{\mathrm{w}}$ is

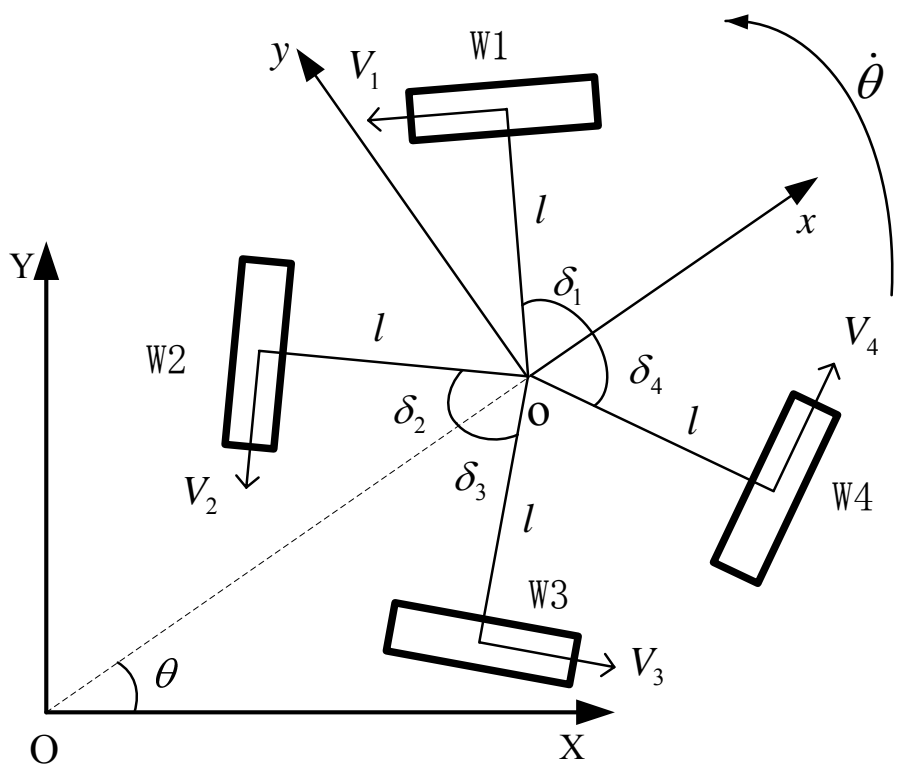

Fig. 1. Wheel placement of a mobile robot

$$
X_{\mathrm{m}}=R(\theta) \cdot X_{\mathrm{w}}=\left[\begin{array}{ccc}
\cos \theta & -\sin \theta & 0 \\
\sin \theta & \cos \theta & 0 \\
0 & 0 & 1
\end{array}\right] . X_{\mathrm{w}},
$$

where $X O Y$ is the world coordinate frame for the robot, xoy is the robot own coordinate frame, $W_{i}, i=1,2,3,4$, denotes the moving direction of the robot, denoting every wheel, $\delta_{i}$ denotes the angle between the wheel and axis respectively, $V_{i}$ denotes the velocity of each wheel, its positive direction is anticlockwise, $l$ is the distance between the center of the robot body and that of each wheel.

The kinematic model of a mobile robot can be constructed as 


$$
\left(\begin{array}{l}
v_{1} \\
v_{2} \\
v_{3} \\
v_{4}
\end{array}\right)=r \cdot\left(\begin{array}{l}
\omega_{1} \\
\omega_{2} \\
\omega_{3} \\
\omega_{4}
\end{array}\right)=\left(\begin{array}{rrr}
-\sin \left(\delta_{1}+\theta\right) & \cos \left(\delta_{1}+\theta\right) & l \\
-\sin \left(\delta_{2}-\theta\right) & -\cos \left(\delta_{2}-\theta\right) & l \\
\sin \left(\delta_{3}+\theta\right) & -\cos \left(\delta_{3}+\theta\right) & l \\
\sin \left(\delta_{4}-\theta\right) & \cos \left(\delta_{4}-\theta\right) & l
\end{array}\right)\left(\begin{array}{c}
\dot{X} \\
\dot{Y} \\
\dot{\theta}
\end{array}\right),
$$

where $v_{i}$ denotes the linear velocity and $\omega_{i}$ is the angular velocity of each wheel, $r$ is the radius of each wheel,

$$
g(\theta)=\left(\begin{array}{rrr}
-\sin \left(\delta_{1}+\theta\right) & \cos \left(\delta_{1}+\theta\right) & l \\
-\sin \left(\delta_{2}-\theta\right) & -\cos \left(\delta_{2}-\theta\right) & l \\
\sin \left(\delta_{3}+\theta\right) & -\cos \left(\delta_{3}+\theta\right) & l \\
\sin \left(\delta_{4}-\theta\right) & \cos \left(\delta_{4}-\theta\right) & l
\end{array}\right) .
$$

\subsection{A dynamic model}

To deal with the model uncertainties and exogenous disturbances of the four-wheel omni-directional mobile robot, firstly we must derive its dynamic equations from the drive motor model.

By applying the Newton's second law in the robot coordinate frame, the force acting on the robot can be derived as

$$
M \ddot{X}_{\mathrm{w}}=g^{\mathrm{T}}(\theta) \cdot f,
$$

where $M=\operatorname{diag}\{m, m, J\}$ is the total mass of the robot, $J$ is the total inertia for the robot rotation, and $f=\left(\begin{array}{lllll}f_{1} & f_{2} & f_{3} & f_{4}\end{array}\right)^{\mathrm{T}}$ is the tangential force generated by DC motors at each wheel.

The dynamics of the armature current of each DC motor can be described by

$$
L_{\mathrm{a}} \frac{d i_{\mathrm{a}}}{d t}+R_{\mathrm{a}} i_{\mathrm{a}}=V_{\mathrm{s}} u-K_{\mathrm{b}} n \dot{\varphi},
$$

where $V_{\mathrm{s}}$ is the battery voltage, $u \in[-1,1]$ is the normalized control input and $L_{\mathrm{a}}$ is the reactance of the motor, $R_{\mathrm{a}}$ is the armature resistance, $K_{\mathrm{b}}$ is the back-emf constant, $n$ is the gear ratio and $\varphi$ is the angular of each wheel.

Since the electrical time constant of the motor is very small compared to the mechanical time constant, we can neglect the inductance of the motor electric circuit and describe the generated torque of each motor $\tau$ as

$$
\tau=K_{\mathrm{t}} n i_{\mathrm{a}}=\frac{1}{R_{\mathrm{a}}} K_{\mathrm{t}} n\left(V_{\mathrm{s}} u-K_{\mathrm{b}} n \dot{\varphi}\right),
$$

where $K_{\mathrm{t}}$ is the torque constant.

The dynamic equation of the velocity for each wheel is

$$
J_{\mathrm{w}} \ddot{\varphi}_{i}+F_{\mathrm{v}} \dot{\varphi}_{i}=\tau_{i}-r f_{i},
$$


where $J_{\mathrm{w}}$ is the inertia at the center of the wheel about the vertical axis and $F_{\mathrm{v}}$ is the viscous friction factor in the drive line.

Then the dynamic formulation produces the system representation, which is described by

$$
D \ddot{q}+C(\dot{q}) \dot{q}=B \tau,
$$

where $q=X_{\mathrm{w}}, \tau=\left(\begin{array}{llll}\tau_{1} & \tau_{2} & \tau_{3} & \tau_{4}\end{array}\right)^{\mathrm{T}}, B=R(\theta) g^{\mathrm{T}}(0)$,

$$
\begin{gathered}
D=\left[\begin{array}{ccc}
\frac{3 J_{\mathrm{w}}}{2 r^{2}}+m & 0 & 0 \\
0 & \frac{5 J_{\mathrm{w}}}{2 r^{2}}+m & \frac{J_{\mathrm{w}}}{r^{2}}(\sqrt{2}-\sqrt{3}) l \\
0 & \frac{J_{\mathrm{w}}}{r^{2}}(\sqrt{2}-\sqrt{3}) l & \frac{4 J_{\mathrm{w}} l^{2}}{r^{2}}+J
\end{array}\right], \text { and } \\
C(\dot{q})=\left[\begin{array}{ccc}
\frac{3}{2}-\frac{J_{\mathrm{w}} \dot{\theta}}{2 r^{2}} \sin 2 \theta & -\dot{\theta}\left(m+\frac{4 J_{\mathrm{w}}}{r^{2}}\right) & 0 \\
\dot{\theta}\left(m+\frac{4 J_{\mathrm{w}}}{r^{2}}\right) & \frac{5}{2}+\frac{J_{\mathrm{w}} \dot{\theta}}{2 r^{2}} \sin 2 \theta & (\sqrt{2}-\sqrt{3}) l \\
\frac{J_{\mathrm{w}} \dot{\theta}}{r^{2}}(\sqrt{2}-\sqrt{3}) l \cos \theta & (\sqrt{2}-\sqrt{3}) l\left(\frac{J_{\mathrm{w}} \dot{\theta}}{r^{2}} \sin \theta+1\right) & 4 l^{2}
\end{array}\right] .
\end{gathered}
$$

For describing conveniently and computing simply, the dynamic equations with disturbances can be rewritten in the standard form

$$
D \ddot{q}+C(\dot{q}) \dot{q}+E(t)=T,
$$

where the virtual control is $T=B \tau$, and $E(t)$ is the total parameter of uncertainties and exogenous disturbances, which is bounded.

\section{Sliding mode control based on an adaptive approach and a filter}

\subsection{Sliding mode control law based on a filter}

According to the theory of the sliding mode control and a filter, if a low pass filter is added to the output port of the variable structure controller, whose output is the input of the robot, then the inherent buffeting caused by the variable structure control may be reduced or eliminated. Considering of the low pass filter is expressed by

$$
Q(s)=\frac{\lambda_{i}}{s_{i}+\lambda_{i}},
$$

where $\lambda_{i}>0, i=1,2, \cdots, k, k$ is the number of the control input $T$. 
According to formulas (8) and (9), belonging to the low pass filter and the virtual control, the relationship between the outputs and inputs is as follows:

$$
\dot{T}+A T=A u,
$$

where $A=\operatorname{diag}\left\{\lambda_{1}, \lambda_{2}, \cdots, \lambda_{k}\right\}$.

Introducing (8) into (10), we obtain

$$
D \dddot{q}+C(\dot{q}) \ddot{q}+\dot{C}(\dot{q}) \dot{q}+\dot{E}+A D \ddot{q}+A C(\dot{q}) \dot{q}+A E=A u .
$$

Supposing that there is a feasible smooth bounded reference trajectory $q_{\mathrm{d}}(t)$, and the tracking error is $e(t)=q(t)-q_{\mathrm{d}}(t)$, for all trajectories starting at $q\left(t_{0}\right)=q_{\mathrm{d}}\left(t_{0}\right)$ we have designed the sliding mode switching function

$$
s(t)=\ddot{e}+A_{1} \dot{e}+A_{2} e,
$$

where $A_{i}=\operatorname{diag}\left\{\lambda_{i 1}, \lambda_{i 2}, \cdots, \lambda_{i k}\right\}, \lambda_{i j}>0, i=1,2, j=1,2, \ldots, k$.

Now choosing the following Lyapunov function candidate as

$$
V=\frac{1}{2} s^{\mathrm{T}} D s
$$

the time derivative of Lyapunov function is

$$
\dot{V}=\frac{1}{2}\left(\dot{s}^{\mathrm{T}} D s+s^{\mathrm{T}} D \dot{s}\right) .
$$

In (11) $D$ is a constant symmetric, positive definite matrix, then $\dot{s}^{\mathrm{T}} D s=s^{\mathrm{T}} D \dot{s}$. Equation (14) can be rewritten as

$$
\begin{gathered}
\dot{V}=s^{\mathrm{T}}\left[D\left(\dddot{q}-\dddot{q}_{d}+A_{1} \ddot{e}+A_{2} \dot{e}\right)\right]= \\
=s^{\mathrm{T}}\left[A u+D\left(A_{1} \ddot{e}+A_{2} \dot{e}-\dddot{q}_{d}\right)-\right. \\
-(A D+C(\dot{q})) \ddot{q}-(A C(\dot{q})+\dot{C}(\dot{q})) \dot{q}-A E(t)-\dot{E}(t)]= \\
=s^{\mathrm{T}}(A u+H),
\end{gathered}
$$

where $H=D\left(A_{1} \ddot{e}+A_{2} \dot{e}-\dddot{q}_{d}\right)-(A D+C(\dot{q})) \ddot{q}-(A C(\dot{q})+\dot{C}(\dot{q})) \dot{q}-A E(t)-\dot{E}(t)$.

By the sliding mode and Lyapunov theory, if the closed-loop system is stable, the control law is

$$
u=A^{-1} \dot{T}+T=-A^{-1}[H+\eta \operatorname{sgn}(s)],
$$

where $\eta$ is the switch gain, then from (15) and (16) we have

$$
\dot{V}=-s^{\mathrm{T}} \eta \operatorname{sgn}(s)=-\eta|s| \leq 0 .
$$

The control law (16) is the variable structure control law based on a filter. By the variable structure control theory, it means that considering system (8) in a closed loop with the control law in (16), then the tracking error $e(t)$ is globally 
asymptotically stabilized to zero. However, the parameters of $H$ must have been confirmed before the control law is applied. If the parameters are not exactly known or changed for some reasons, the control performance might get worse.

\subsection{Adaptive approach}

As above described, the controller, whose representation is given in (16), is applied usually by the expression of $H$ exactly known. However, this is impossible in practical application.

Defining the estimated value of $H$ as $\hat{H}$, using that instead of $H$, the estimated error is $\tilde{H}=H-\hat{H}$, and assuming that the parameters of $H$ are slow time variables, we have the new Lyapunov function candidate as follows:

$$
V_{1}=\frac{1}{2} s^{\mathrm{T}} D s+\frac{1}{2 \sigma} \tilde{H}^{\mathrm{T}} \tilde{H}, \sigma>0 .
$$

Similarly, the time derivative is given by

$$
\dot{V}_{1}=s^{\mathrm{T}}(A u+H)-\frac{1}{\sigma} \tilde{H}^{\mathrm{T}} \dot{\hat{H}}=s^{\mathrm{T}}(A u+\hat{H})+\tilde{H}^{\mathrm{T}} s-\frac{1}{\sigma} \tilde{H}^{\mathrm{T}} \dot{\hat{H}} .
$$

According to the sliding mode theory, if we choose the new control law as

$$
u=-A^{-1}[\hat{H}+\eta \operatorname{sgn}(s)]
$$

and the adapting law of $\hat{H}$ is

$$
\dot{\hat{H}}=\sigma \cdot s,
$$

then we have

$$
\dot{V}_{1}=-s^{\mathrm{T}} \eta \operatorname{sgn}(s)=-\eta|s| \leq 0 .
$$

In the same way, it means that considering system (8) in a closed loop with the new adaptive controller (20) and the adapting law of $\hat{H}$ in (21), then the tracking error $e(t)$ is globally asymptotically stabilized to zero, even when the parameters are not exactly confirmed.

\subsection{RBF based reaching law sliding mode control}

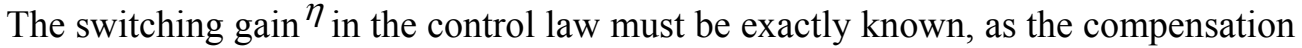
of the boundness of the model uncertainties and exogenous disturbances is usually bigger and may cause high noise amplification and high control cost, particularly for the complex dynamics of the omni-directional mobile manipulator system. Therefore, in order to improve the dynamic quality and to raise the speed of reaching the sliding surface $s=0$, the reaching law is presented as

$$
\dot{s}=-\eta_{1} \operatorname{sgn}(s)-\varepsilon s,
$$


where the constants $\eta_{1}>0$ and $\varepsilon>0$.

Taking in mind equations (11), (12), (20) and (21) into (23), we have the new control law

$$
u=-A^{-1}\left[\hat{H}+D\left(\eta_{1} \operatorname{sgn}(s)+\varepsilon s\right)\right] .
$$

The control law can be rewritten as two parts

$$
u=u_{\mathrm{eq}}+u_{\mathrm{sw}}
$$

where $u_{\text {eq }}=-A^{-1} \hat{H}, u_{\mathrm{sw}}=-A^{-1}(\eta \operatorname{sgn}(s)+\kappa \mathrm{s}), \eta=D \eta_{1}, \kappa=D \varepsilon$.

The reaching control part $u_{\mathrm{sw}}$ is very important for the performance of the whole control system, a Radial Basis Function based Neural Network (RBFNN) is used to identify and approach the reaching gains $\eta$ and $\kappa$. Using the learning ability of RBFNN, it can co-ordinately control the omni-directional mobile robot with different dynamics efficiently. By taking the sliding mode switching function $s$ as an input of RBFNN, and the reaching gains as its outputs, taking $\eta$ as an example, we obtain

$$
\eta=|W R(s)|
$$

where $R(s)=\exp \left(-\|s-c\|^{2} / b^{2}\right)$ is the Gauss function, $W$ is a matrix of weights between the hidden layer and the output layer, $c$ is the center vector and $b$ is the width vector of the Radial Basis Function.

The weight adapting performance index is $J_{\mathrm{p}}=s(t) \dot{s}(t)$, then the net parameters are

$$
\begin{aligned}
& W(t+1)=W(t)+\Delta W+\alpha(W(t)-W(t-1)), \\
& c(t+1)=c(t)+\Delta c+\alpha(c(t)-c(t-1)), \\
& b(t+1)=b(t)+\Delta b+\alpha(b(t)-b(t-1)),
\end{aligned}
$$

where the constant $\alpha \in(0,1)$ is the inertial ratio.

The weight-updating equations are:

$$
\begin{gathered}
\Delta W=-\mu_{\mathrm{w}} \frac{\partial s \dot{s}}{\partial u} \frac{\partial u}{\partial u_{\mathrm{sw}}} \frac{\partial u_{\mathrm{sw}}}{\partial \eta} \frac{\partial \eta}{\partial W}, \\
\Delta c=-\mu_{\mathrm{c}} \frac{\partial J_{\mathrm{p}}}{\partial c}=-\mu_{\mathrm{c}} \frac{\partial s \dot{s}}{\partial c} \frac{\partial u}{\partial u_{\mathrm{sw}}} \frac{\partial u_{\mathrm{sw}}}{\partial \eta} \frac{\partial \eta}{\partial R} \frac{\partial R}{\partial c}, \\
\Delta b=-\mu_{\mathrm{b}} \frac{\partial J_{\mathrm{p}}}{\partial b}=-\mu_{\mathrm{b}} \frac{\partial s \dot{s}}{\partial b} \frac{\partial u}{\partial u_{\mathrm{sw}}} \frac{\partial u_{\mathrm{sw}}}{\partial \eta} \frac{\partial \eta}{\partial R} \frac{\partial R}{\partial b}, \\
\frac{\partial u}{\partial u_{\mathrm{sw}}}=1, \frac{\partial u_{\mathrm{sw}}}{\partial \eta}=A^{-1} D \operatorname{sgn}(R), \\
\frac{\partial \eta}{\partial W}=W \operatorname{sgn}(R), \frac{\partial R}{\partial c}=R \frac{s-c}{b^{2}}, \frac{\partial R}{\partial b}=R \frac{(s-c)^{2}}{b^{3}} .
\end{gathered}
$$




\section{Numerical simulation}

To assess the performance of our controller, some numerical simulations are carried out tracking a straight path and a sinusoidal path separately. The values of the parameters correspond to a laboratory prototype built in our institution and they are presented in Table 1.

Table 1. Parament values of the robot

\begin{tabular}{|c|c|c|c|}
\hline Parameters & Values & Parameters & Values \\
\hline$F_{\mathrm{v}}$ & $1.86(\mathrm{~N} \cdot \mathrm{m}) /(\mathrm{rad} / \mathrm{s})$ & $J_{\mathrm{w}}$ & $0.8 \mathrm{~g} . \mathrm{cm}^{2}$ \\
\hline$K_{\mathrm{t}}$ & $0.0259(\mathrm{~N} \cdot \mathrm{m}) / \mathrm{A}$ & $V_{\mathrm{s}}$ & $24 \mathrm{~V}$ \\
\hline$K_{\mathrm{b}}$ & $0.0259 \mathrm{~V} /(\mathrm{rad} / \mathrm{s})$ & $m$ & $23 \mathrm{~kg}$ \\
\hline$n$ & 22 & $L$ & $0.225 \mathrm{~m}$ \\
\hline$R_{\mathrm{a}}$ & $0.611 \Omega$ & $r$ & $0.1 \mathrm{~m}$ \\
\hline$J$ & $33.3 \mathrm{~g} \mathrm{~cm}^{2}$ & & \\
\hline
\end{tabular}

In the numerical simulations, supposing that the total disturbances and uncertainties in the tracking process are

$$
E(t)=[2 \sin (2 \pi t) 1.5 \cos (2 \pi t) \quad 3 \sin (2 \pi t)]^{\mathrm{T}},
$$

the initial posture is $q_{d}(0)=\left[\begin{array}{lll}0.5 & 0.5 & 0.5\end{array}\right]^{\mathrm{T}}$.

The learning ratios of RBFNN are $m_{\mathrm{w}}=0.05, m_{\mathrm{c}}=0.3, m_{\mathrm{b}}=0.3, \alpha=0.05$, the initial values of the parameters are $W=\left[\begin{array}{lll}0.25 & 0.25 & 0.25 \\ 0.25 & 0.25 & 0.25\end{array}\right], \quad c=3$, $b=\left[\begin{array}{lll}0.5 & 0.5 & 0.5\end{array}\right]^{\mathrm{T}}$. The adapting weight is $\sigma=30$, and the matrixes

$$
\begin{aligned}
A_{1}=\operatorname{diag}\{15,15,15\}, A_{2} & =\operatorname{diag}\{45,45,45\}, A=\operatorname{diag}\{3.5,3.5,3.5\}, \\
\eta & =\operatorname{diag}\{41,94,50\} .
\end{aligned}
$$

Firstly, we let the robot to track a straight path, when the parameters in Table 1 are exactly known, the trajectory tracking results and the practical control inputs on $X$ axis are shown in Fig. 2 (a) and Fig. 2 (b). When the parameter $m$ in Table 1 is changed from $23 \mathrm{~kg}$ to $12 \mathrm{~kg}$, the results of the trajectory tracking and the torque signals in $X$ axis are shown in Fig. 2 (c) and Fig. 2 (d). The left column figures in Fig. 2 show the results considering the robust Neural Network based Sliding Mode Controller (NNSMC) in [13] and the right column figures are connected with the control strategy proposed in this paper (AFSMC). The numeric comparison between NNSMC and AFSMC is given in Table 2.

Table 2. Numeric comparison when tracking a straight path

\begin{tabular}{|c|c|c|c|c|}
\hline Time & Approaches & $\begin{array}{c}\text { Tracking } \\
\text { time (s) }\end{array}$ & $\begin{array}{c}\text { Means of } \\
\text { input }(\mathrm{N})\end{array}$ & $\begin{array}{c}\text { Variances of } \\
\text { input }\end{array}$ \\
\hline \multirow{2}{*}{ Before $m$ changed } & NNSMC & 1.65 & 0.2745 & 3.2447 \\
\cline { 2 - 5 } & AFSMC & 1.20 & 0.0664 & 1.2142 \\
\hline \multirow{2}{*}{ After $m$ changed } & NNSMC & $\infty$ & 0.1583 & 2.1153 \\
\cline { 2 - 5 } & AFSMC & 1.43 & -0.0791 & 1.2598 \\
\hline
\end{tabular}



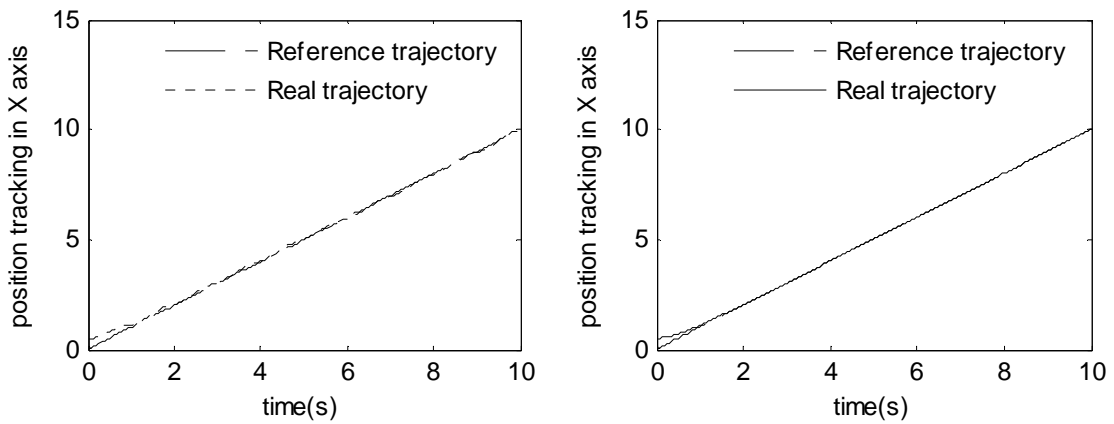

(a) Trajectory tracking results with exact parameters
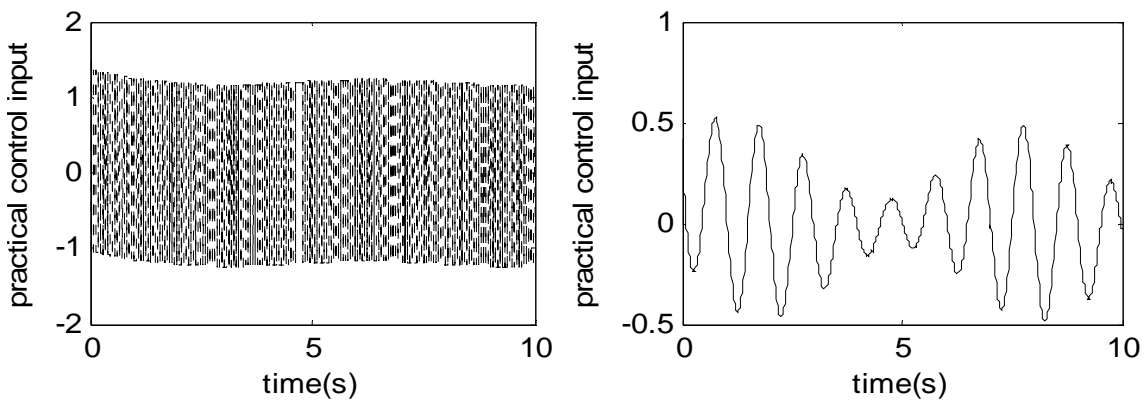

(b) Control torque signals on $X$ axis with exact parameters
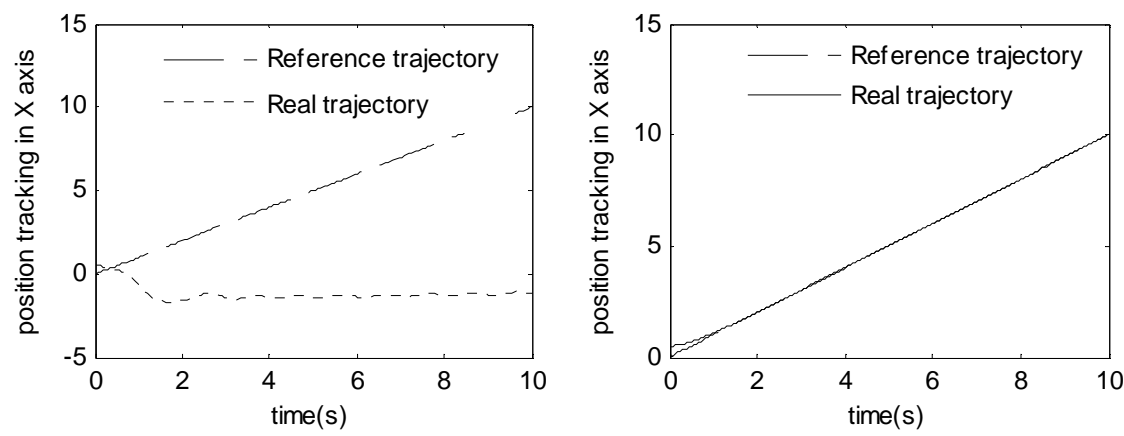

(c) Trajectory tracking results on $X$ axis when $m$ is changed
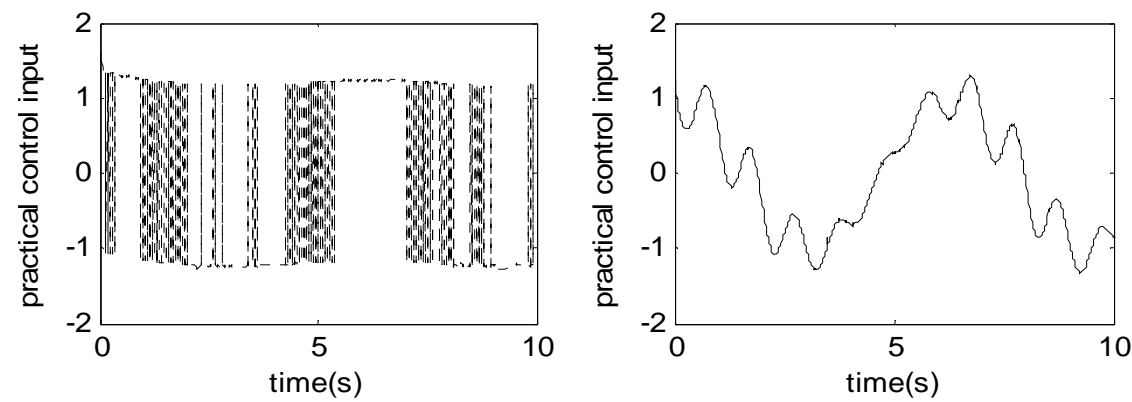

(d) Control torque signals on $X$ axis when $m$ is changed

Fig. 2. Simulation results for tracking a straight path 
From Table 2 and Fig. 2 (a), when the parameters are exactly confirmed, NNSMC can make the robot tracking the path quickly with a smaller tracking error, and the tracking time of AFSMC is 1.20 s, which is smaller than 1.65s . But in Fig. 2 (b) there is strenuous vibration of the control input with NNSMC, the variance of the input is 3.2447 , which is 2.6 times more than that of AFSMC. When the parameters are not exactly confirmed, $m$ is changed from $23 \mathrm{~kg}$ to $12 \mathrm{~kg}$, in Fig. 2 (c) and Fig.2 (d), NNSMC can not track the reference path quickly, and the tracking errors become bigger and bigger, and its control input is shaken acutely. However, AFSMC can still make the robot track its desired path with good performance and smooth input, because the adaptive approach, RBFNN and a filter can eliminate the chatter efficiently.

In order to test the performance when the path is variable, we let the robot track a sinusoidal path, the tracking results are shown in Fig. 3. The left column figures in Fig. 3 show the results when NNSMC is considered and the right column figures are for the control strategy AFSMC. The numeric comparison between NNSMC and AFSMC is given in Table 3.

Table 3. Numeric comparison when tracking a sinusoidal path

\begin{tabular}{|l|c|c|c|c|}
\hline & Approaches & $\begin{array}{c}\text { Tracking } \\
\text { time(sec) }\end{array}$ & $\begin{array}{c}\text { Means of } \\
\text { input(N) }\end{array}$ & $\begin{array}{c}\text { Variances of } \\
\text { input }\end{array}$ \\
\hline \multirow{3}{*}{ Before $m$ changed } & NNSMC & 2.25 & -1.2043 & 3.2447 \\
\cline { 2 - 5 } & AFSMC & 1.35 & 0.5580 & 1.1933 \\
\hline \multirow{3}{*}{ After $m$ changed } & NNSMC & $\infty$ & -0.3014 & 2.4094 \\
\cline { 2 - 5 } & AFSMC & 1.50 & -0.3593 & 1.3220 \\
\hline
\end{tabular}

When the parameters are exactly confirmed, from Table 3 and Fig. 3 (a) it follows that AFSMC can make the robot track the path quickly with a smaller tracking error, but the response speed is slower at the initial time when using the NNSMC. In Fig. 3 (b) the control input with NNSMC is drastically shaken, but the input with AFSMC, except for a bit of buffeting, is smoother. It is because the chatter can be efficiently reduced by AFSMC. When the parameters are not exactly confirmed, from Fig. 3(c) and Fig. 3 (d) is obvious that NNSMC cannot track the reference path, it even loses the desired path, and its input is still not smooth. However, AFSMC can make the robot track its desired path with good performance, and Table 3 shows that the variance of the input is only 1.3220 , that is $54.9 \%$ of that with NNSMC.

In conclusion, as shown in Figs 2 and 3, the trajectory tracking results have shown that the properties of the closed-loop system have better performance than the control obtained by NNSMC, whether or not the parameter is constant or changed. The inherent buffeting has been reduced efficiently when AFSMC is considered. However, NNSMC has caused high noise amplifications and high control costs. 

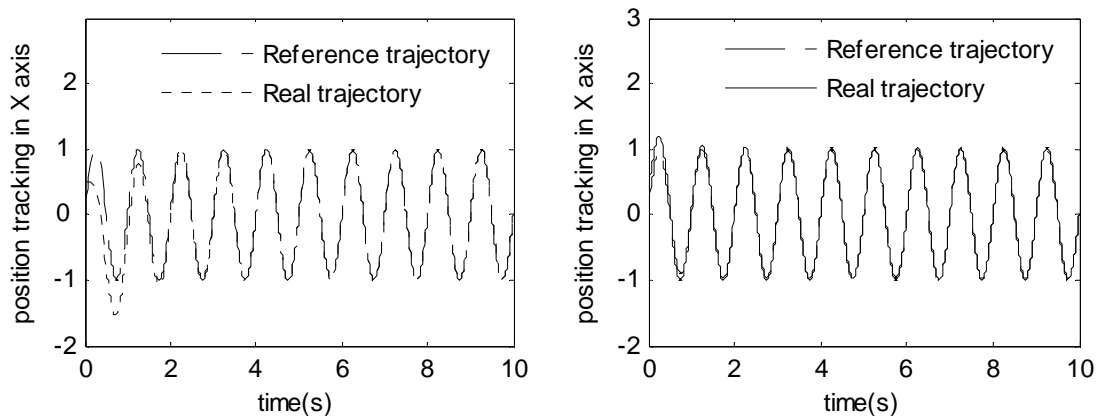

(a) Trajectory tracking results with exact parameters
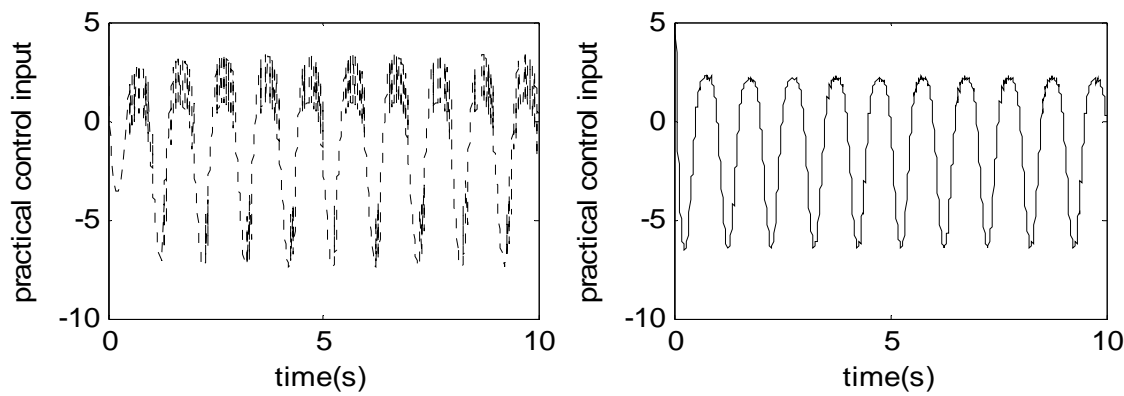

(b) Control torque signals on $X$ axis with exact parameters
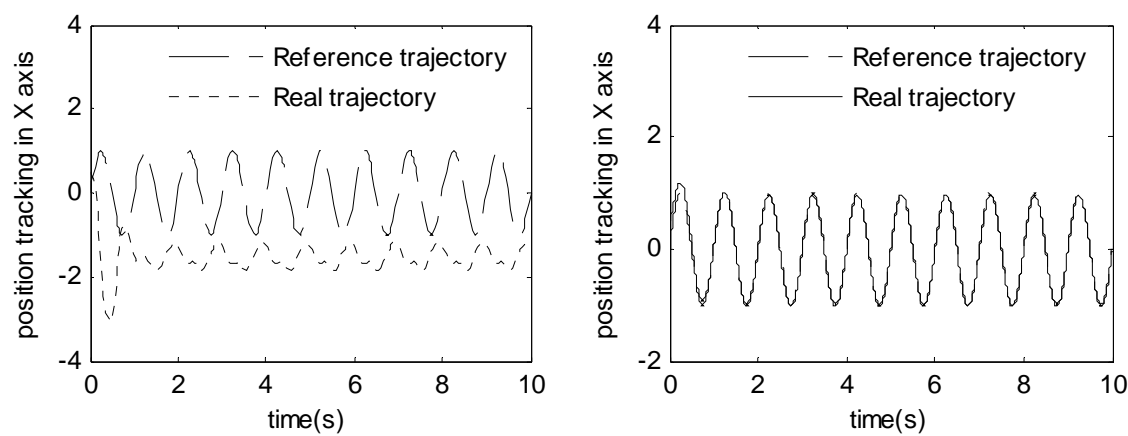

(c) Trajectory tracking results on $X$ axis when $m$ is changed
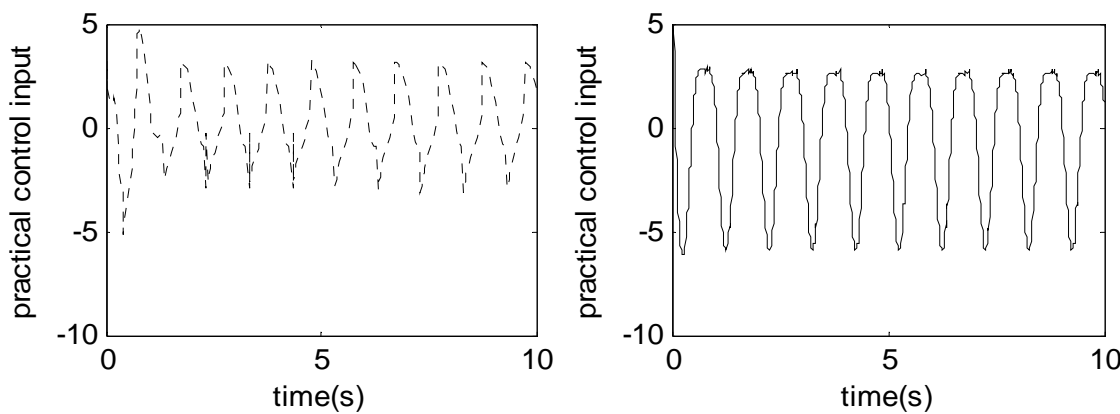

(d) Control torque signals on $X$ axis when $m$ is changed

Fig. 3. Simulation results for tracking a sinusoidal path 


\section{Conclusion}

The motion control with a model of a four-wheel omni-directional mobile robot considering its dynamic model uncertainties and exogenous disturbances, has been addressed and solved by means of an adaptive variable structure control based on a filter. The asymptotic stability of the closed loop system has been formally proved. Numerical simulations have been proposed to illustrate the properties of the closedloop system showing a better performance than the control obtained by NNSMC. It has shown that the control system with AFSMC has better tracking performance of the motion control and the results have shown that this method has good tracking robustness and high control precision, simple achievement and efficiently eliminated buffeting.

Acknowledgements: The work of this paper was supported by the Natural Science Fund Free Application Project of Guangdong Province (No S2011010004006), the Industry-university-institute Cooperation Project of Guangdong Province and Chinese Ministry of Education (No 2012B091100423), the Science and Technology Planning Project of Zhaoqing City (No 2010F006, No 2011F001), and the Research Initiation Fund of Zhaoqing University (No 2012BS01).

\section{References}

1. J e fri, E. M. S., R. Moh a med, Y. S a z a 1 i. Designing Omni-Directional Mobile Robot with Mecanum Wheel. - American Journal of Applied Sciences, Vol. 3, 2006, No 5, 1831-1835.

2. L i u, Y., X. W u, J. Z h u, et al. Omni-Directional Mobile Robot Controller Design by Trajectory Linearization. - In: Proceedings of 2003 American Control Conference, Vol. 4, 2003, 3423-3428.

3. Y a n g, S. X., A. Zhu, G. F. Yu a n et al. A Bioinspired Neurodynamics Based Approach to Tracking Control of Mobile Robots. - IEEE Transactions on Industrial Electronics, Vol. 59, 2012, No 8, 3211-3220.

4. K a $1 \mathrm{~m}$ a r-N a g y, T., D. A. R a ffa e 11 o, P. G a ng gu ly. Near-Optimal Dynamic Trajectory Generation and Control of an Omnidirectional Vehicle. - Robotics and Autonomous System, Vol. 46, 2004, 47-64.

5. Cu i, Q. Z., X. Li, X. K. W ang et al. Backstepping Control Design on the Dynamics of the Omni-Directional Mobile Robot. - Applied Mechanics and Materials, Vol. 203, 2012, 51-56.

6. Ch e n, S. H., J. C. J u a n g, S. H. S u. Backstepping Control with Sum of Squares Design for Omni-Directional Mobile Robots. - In: Proceedings of ICCAS-SICE, 2009, 545-550.

7. Purwin, O., R. D. Andrea. Trajectory Generation and Control for Four Wheeled Omnidirectional Vehicles. - Robotics and Autonomous System, Vol. 54, 2006, 13-22.

8. Y a n g, H., S. X. Y a n g, G. S. M i t t a l. Tracking Control of a Nonholonomic Mobile Robot by Integrating Feedback and Neural Dynamics Techniques. - In: Proceedings of 2003 IEEE/RSJ International Conference on Intelligent Robots and Systems, Vol. 4, 2003, 3522-3527.

9. Y a n g, J. Y., D. C. B a i, S. Y. W a n g, et al. Trajectory Tracking Control of Omni-Directional Wheeled Robot for Lower Limbs Rehabilitative Training. - ROBOT, Vol. 33, 2011, No 3, 314-318.

10. Willi a m s, R. L., B. E. Carter, P. Gallina, et al. Dynamic Model with Slip for Wheeled Omnidirectional Robots. - IEEE Transactions on Robotics and Automation, Vol. 18, 2002, No 3, 285-293.

11. S u n, B., D. Q. Z h u, Z. G. D e n g. Bio-Inspired Discrete Trajectory-Tracking Control for OpenFrame Underwater Vehicles. - Control Theory \& Applications, Vol. 30, 2013, No 4, 454-462. 
12. S h o-T s u n g, K., C. W a n-J u n g, H. M i n g-T z u. Integral Sliding Mode Control for Trajectory Tracking Control of an Omnidirectional Mobile Robot. - In: 8th Asian Control Conference (ASCC), 2011, 765-770.

13. X u, D., D. B. Z h a o, J. Y. Y i, X. M. T a n. Trajectory Tracking Control of Omnidirectional Wheeled Mobile Manipulators: Robust Neural Network-Based Sliding Mode Approach. IEEE Transactions on Systems, Man, and Cybernetics, Part B: Cybernetics, Vol. 39, 2009, No 4, 788-799.

14. C a o, Z. C., Y. T. Z h a o, Q. D. W u. Point Stabilization of a Nonholonomic Mobile Robot Based on Backstepping And Neural Dynamics. - Acta Electronica Sinica, Vol. 39, 2011, No 3, 591-595. 